\title{
Cracking due to shear in masonry mortar joints and around the interface between masonry walls and reinforced concrete beams
}

\author{
José Luís Miranda Dias * \\ National Civil Engineering Laboratory, Buildings Department, Av. do Brasil, 101, Lisboa 1700-066, Portugal
}

Received 15 December 2004; received in revised form 26 June 2005; accepted 8 July 2005

Available online 27 September 2005

\begin{abstract}
Cracking occurs frequently in building masonry walls, and are usually located in the joints between the units because they represent generally planes of weakness, in some cases due to their low shear resistance. Sometimes cracks can appear in the interface between masonry blocks and reinforced concrete beams. That cracking has negative implications in building performance, namely, due to the possibility of rain penetration through the cracks and to negative aesthetic aspect. So, the objective of this paper is essentially to achieve a better knowledge of the shear behaviour of joints in masonry, for the particular cases of solid lightweight concrete blocks (blocks of aerated autoclaved concrete and blocks of lightweight concrete with expanded clay aggregates) and solid clay bricks, and to find preventive solutions to minimize those defects.

This paper presents the principal results and conclusions of an experimental study that concerned the determination of mortar joints shear behaviour. From the results of the experimental tests, it can be concluded that the properties of the referred joints are significant for the wall overall behaviour, and the inclusion of reinforcing elements in these joints may in some cases serve as a preventive measure and can determine positive implications on that behaviour.
\end{abstract}

(C) 2005 Elsevier Ltd. All rights reserved.

Keywords: Masonry walls; Buildings; Shear; AAC; Lightweight concrete

\section{Introduction}

Movements of masonry walls confining elements (such as reinforced concrete beams, slabs, roofs and foundations), caused by excessive deflections, settlements or thermal effects [5-7], can generate stress in the confined walls, which result in cracks in the masonry, especially in their mortar joints frequently due to shear effects. In fact, these joints represent generally planes of weakness, in some cases due to their low shear resistance [9]. That cracking has negative implications in building performance, namely, due to the possibility of rain penetration through the cracks and to negative aesthetic aspect. So, the objective of this paper is essentially to achieve a better knowledge of the shear behaviour of masonry wall joints (common unit/unit joint),

\footnotetext{
${ }^{*}$ Tel.: +351 218443663.

E-mail address: mirandadias@lnec.pt.
}

for the particular cases of solid lightweight concrete blocks (blocks of aerated autoclaved concrete - material X, with dry mass of around $560 \mathrm{~kg} / \mathrm{m}^{3}$; blocks of lightweight concrete with expanded clay aggregates - mater. Y, with dry mass of around $1040 \mathrm{~kg} / \mathrm{m}^{3}$ ) and solid clay bricks (material $\mathrm{Z}$ with dry mass of around $2110 \mathrm{~kg} / \mathrm{m}^{3}$ ).

Here, mention is made of the principal results and conclusions of an experimental study, carried out at LNEC, that concerned the determination of mortar joints shear behaviour for the type of blocks and bricks above referred.

\section{General description of shear tests}

\subsection{Initial considerations}

Shear tests were developed as part of an experimental study on masonry walls $[7,8]$. In that study, material properties of the units used for the masonry specimens as well as 
the mortar used in their joints were estimated taking, firstly, into account the results of uniaxial compression tests and flexure tension tests (Table 1). But the mortar joint and the unit properties cannot be merely defined from simple uniaxial and flexure tests, since the state of stress within the joint is complex due to the influence of the surrounding units. Therefore, triaxial compression tests were carried out on mortar samples (for specimens of materials $\mathrm{X}, \mathrm{Y}$ and $\mathrm{Z}$ : thin mortar (X) and cement mortar (Y and $\mathrm{Z})$ ) and on samples made from the material of the three types of units used (see results in Table 1). The samples of materials $\mathrm{X}, \mathrm{Y}$ and $\mathrm{Z}$ were taken, respectively, from blocks of aerated autoclaved concrete, with approximate dimensions of $600 \mathrm{~mm} \times 200 \mathrm{~mm} \times 150 \mathrm{~mm}$ (thickness) and mean moisture content of around 21.5\%; blocks of lightweight concrete with expanded clay aggregates with approximate dimensions of $500 \mathrm{~mm} \times 200 \mathrm{~mm} \times 150 \mathrm{~mm}$ (thickness) and mean moisture content of around $6.8 \%$; and solid clay bricks with approximate dimensions of $229 \mathrm{~mm} \times 73 \mathrm{~mm} \times$ $150 \mathrm{~mm}$ (thickness).

From the triaxial compression (ratio of lateral stress/ vertical stress, $\left(\sigma_{\mathrm{L}} / \sigma_{\mathrm{V}}\right)$, of the specimens ranging generally between 0.05 and 0.30 ) on unit and mortar material, average stress-strain curves were derived and elastic properties were estimated. The strength of the joint under tension was estimated from a series of flexure strength tests on solid blockwork couplets (with a vertical joint in the middle of the specimen). So, the estimated tensile strength was 0.6 and $0.2 \mathrm{MPa}$, respectively for couplets made of material $\mathrm{X}$ and $\mathrm{Y}$, which can be considered a low value of masonry tensile bond strength (no tests were made on material $\mathrm{Z}$ ).

\subsection{Shear test set-up}

Different shear test methods were once reported by researchers for the determination of shear strength of masonry mortar joints $[9,10]$, and their general performance was found to be, basically, dependent on the evenness of the distribution of normal and shear stress in bed joint and the occurrence of undesirable normal stress across the bed joint [11]. Recently, the triplet test was chosen by CEN (CEN, pr EN 1052-3, 1999 [2]) to be the standard test for the evaluation of the shear strength of masonry joints. This test allows, essentially, to obtain the initial shear strength value $-f_{\text {vko }}$. Previous experimental study proposed the use of rock mechanics shear test apparatus for the investigation of shear behavior of mortar joints [3], comparing it in terms of the information about shear bed joint behavior that can be obtained with others types of test apparatus, and the results found with the proposed method were considered satisfactory. This could give some advantages in terms of more information about shear stress and deformation behaviour of the bed joints. So, with a shear test apparatus used in rock mechanics (Fig. 1(a)), several tests on specimens of masonry blocks and bricks were performed [4]. In this set-up, the normal and shear load actuators could operate independently in force mode and transmit to a specimen fixed inside the shear box (as shown in Fig. 1(a)) a constant normal load and, simultaneously, a variable shear load, while the normal displacements in four points and the shear displacements in two points could be monitored by digital transducers (see Figs. 1(a)-(d)). The shear stress across the horizontal bed joint of the specimens used in this study, analyzed by finite element method, were found to be almost uniform with exception to the extreme zones of that joint.

The shear tests were done on specimens (Table 2 block/block joint) made from: blocks of aerated autoclaved concrete (material $\mathrm{X}-\mathrm{Gx}$ and Hx series) and including a thin mortar joint (thickness of $\approx 3 \mathrm{~mm}$ ); blocks of lightweight concrete with expanded clay aggregates (material $\mathrm{Y}-\mathrm{Gy}$ and Hy series) and including a cement mortar joint (thickness of $\approx 5 \mathrm{~mm}$ ); and solid bricks (material $\mathrm{Z}-\mathrm{Gz}$ and $\mathrm{Hz}$ series) and including a cement mortar joint similar to that used for Gy and Hy (thickness of $\approx 5 \mathrm{~mm}$ ). The main difference between $\mathrm{Gx}$, and Hx consisted in the inclusion, in Hx specimens, of a vertical joint to simulate vertical joints in masonry, in addition to the horizontal joint (to simulate bed joints) present in the specimens of Gx series (Table 2). The same considerations could be made about the differences between Gy and $\mathrm{Hy}$ or $\mathrm{Gz}$ and $\mathrm{Hz}$.

Shear tests also were carried out on mixed specimens similar in dimensions to the previous ones (Table $2-$ block/concrete joint), but made from half part of concrete

Table 1

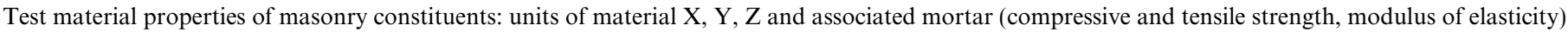

\begin{tabular}{|c|c|c|c|c|c|c|c|c|}
\hline \multirow[t]{2}{*}{ Type of masonry material (units and mortar joints) } & \multicolumn{3}{|c|}{$f_{\mathrm{c}}(\mathrm{MPa})$} & \multicolumn{2}{|c|}{$f_{\mathrm{t}}(\mathrm{MPa})$} & \multicolumn{3}{|c|}{$E(\mathrm{MPa})$} \\
\hline & Block & Mortar & Masonry & Block & Mortar & Block & Mortar & Masonry \\
\hline $\begin{array}{l}\text { Material } X \\
\text { Blocks: of aerated autoclaved concrete } \\
\text { Mortar: thin layer mortar (adhesive type) }\end{array}$ & 3.9 & 5.2 & 2.0 & 1.3 & 2.9 & 930 & 1580 & 1300 \\
\hline $\begin{array}{l}\text { Material } Y \\
\text { Blocks: lightweight concrete with expanded clay aggregates } \\
\text { Mortar: cement, sand (mix proportions }-1: 5 \text { ) }\end{array}$ & 10.1 & 2.6 & 4.5 & 2.7 & 2.1 & 8500 & 1300 & 5070 \\
\hline $\begin{array}{l}\text { Material } Z \\
\text { Bricks: solid clay bricks } \\
\text { Mortar: cement, sand (mix proportions }-1: 5 \text { ) }\end{array}$ & 27.1 & 2.5 & - & 5.5 & 1.4 & 1570 & 1300 & - \\
\hline
\end{tabular}




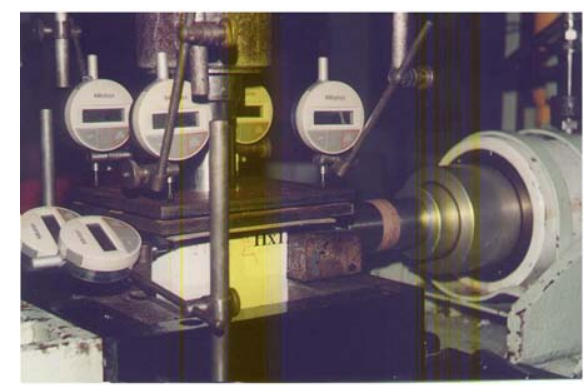

a
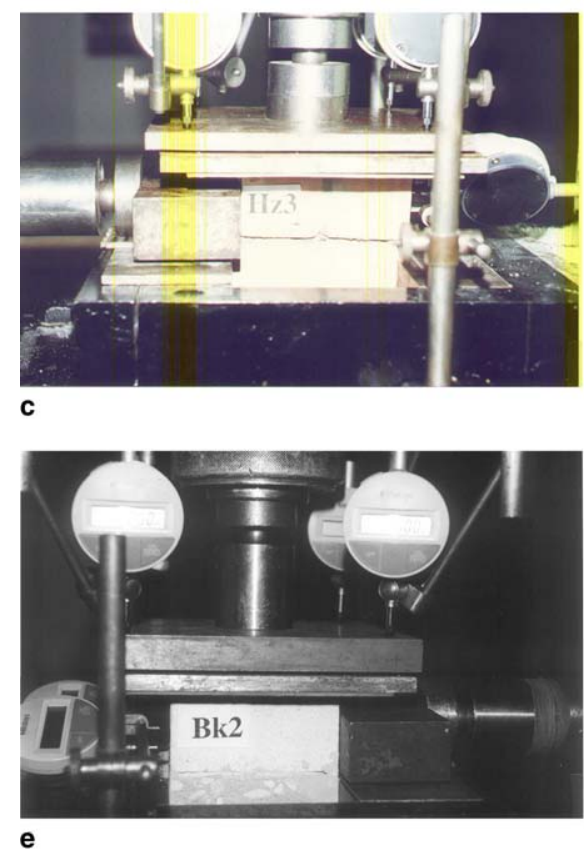

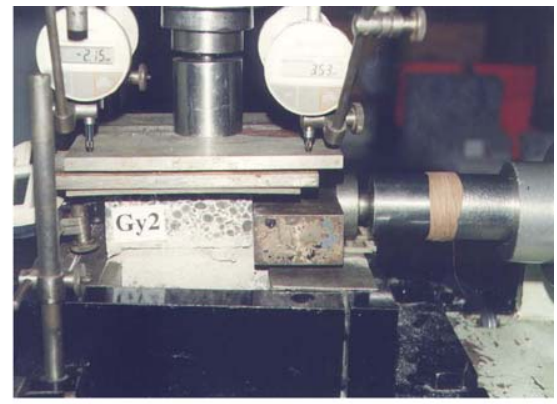

b

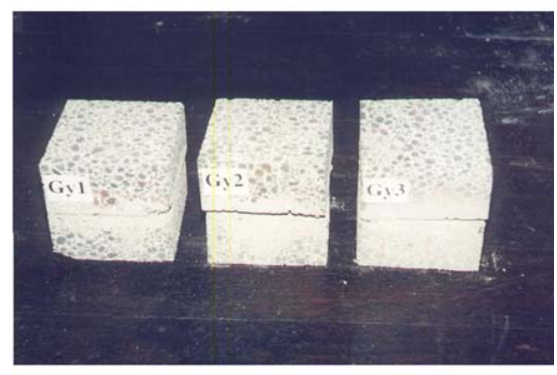

d

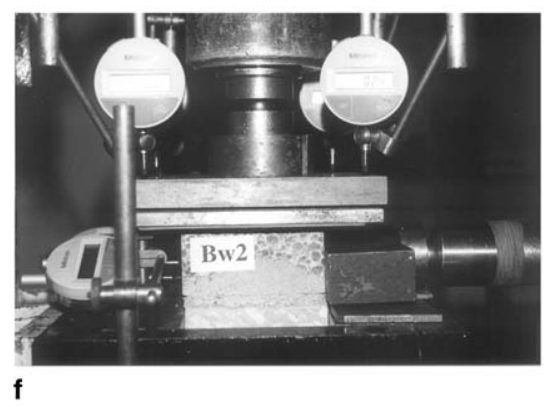

Fig. 1. (a) Instrumentation of the shear test (Hx1 specimen); (b) shear test of Gyl specimen; (c) shear test of Hz3 specimen; (d) final aspect of shear test specimens of Gy series (Gy1, Gy2, Gy3); (e) shear test of Bk2 specimen; (f) shear test of Bw2 specimen.

Table 2

General description of shear tests of specimens

Blocks of aerated autoclaved concrete

Blocks of lightweight concrete with expanded clay aggregates

Solid bricks

Blocks of aerated autoclaved concrete/ normal concrete

Blocks of lightweight concrete with expanded clay aggregates/normal concrete

Solid bricks/normal concrete

Graphic scheme of the specimen
$\mathrm{Gx} 1, \mathrm{Gx} 2, \mathrm{Gx} 3, \mathrm{Gx} 40.50 \mathrm{MPa} \leqslant \sigma_{\mathrm{V}} \leqslant 1.25 \mathrm{MPa}$ Gy1, Gy2, Gy3 0.75 MPa $\leqslant \sigma_{\mathrm{V}} \leqslant 1.25 \mathrm{MPa}$

$\mathrm{Gz} 1, \mathrm{Gz} 2, \mathrm{Gz} 30.75 \mathrm{MPa} \leqslant \sigma_{\mathrm{V}} \leqslant 1.25 \mathrm{MPa}$

$\mathrm{Bk} 1, \mathrm{Bk} 2, \mathrm{Bk} 30.50 \mathrm{MPa} \leqslant \sigma_{\mathrm{V}} \leqslant 1.25 \mathrm{MPa}$

$\mathrm{Bw} 1, \mathrm{Bw} 2, \mathrm{Bw} 30.75 \mathrm{MPa} \leqslant \sigma_{\mathrm{V}} \leqslant 1.25 \mathrm{MPa}$

$\mathrm{Bv} 1, \mathrm{Bv} 2 \sigma_{\mathrm{V}}=0.75 \mathrm{MPa}$

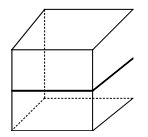

$\mathrm{Hx} 1, \mathrm{Hx} 2, \mathrm{Hx}^{\mathrm{a}}{ }^{0} 0.75 \mathrm{MPa} \leqslant \sigma_{\mathrm{V}} \leqslant 1.25 \mathrm{MPa}$ Hy1, Нy2, Нy $3^{\mathrm{a}} 0.75 \mathrm{MPa} \leqslant \sigma_{\mathrm{V}} \leqslant 1.25 \mathrm{MPa}$

$\mathrm{Hz} 1, \mathrm{~Hz} 2, \mathrm{~Hz}^{\mathrm{a}}{ }^{0} 0.75 \mathrm{MPa} \leqslant \sigma_{\mathrm{V}} \leqslant 1.25 \mathrm{MPa}$

$$
-
$$

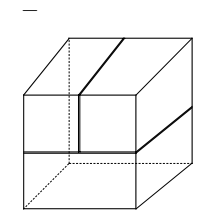

${ }^{\mathrm{a}}$ In these series (Hx, Hy and $\mathrm{Hz}$ ) the shear load was applied normal to the vertical joint of the specimen (see Fig. 1(c)).

(same material as used for reinforced concrete beams done at the same time [4] - concrete with $f_{\mathrm{cm}}=33 \mathrm{MPa}$ and $f_{\text {ctm }}=4 \mathrm{MPa}$ ) and other half part of masonry block mate- rial: blocks of aerated autoclaved concrete (material X - Bk series, see a test of a specimen example in Fig. 1(e)) including a thin mortar joint (thickness of approximately $3 \mathrm{~mm}$ ); 
blocks of lightweight concrete with expanded clay aggregates (material $\mathrm{Y}-\mathrm{Bw}$ series) including a cement mortar joint (thickness of $\approx 5 \mathrm{~mm}$ ); and solid bricks (material $\mathrm{Z}$ $\mathrm{Bv}$ series, see a test of a specimen example in Fig. 1(f)) including a cement mortar joint similar to that used for $\mathrm{Bw}$ (thickness of $\approx 5 \mathrm{~mm}$ ).

The methodology of the shear tests consisted in the initial application, on each specimen (Fig. 1), of a constant normal compressive load (level of load different from specimen to specimen, and that could range between 0.50 and 1.25 MPa - Table 2) by means of a hydraulic jack followed by a gradual application of shear horizontal load, also by hydraulic jack, and by the starting of the normal and shear displacements measurements (shear load-displacements curves).

The applied shear load was gradually increased by steps (generally corresponding to a step increment of shear stress, $\Delta \sigma_{\mathrm{T}}$, of $0.50 \mathrm{MPa}$ ), till the failure of the specimen was reached, while the normal load was maintained permanently constant. The relative peak shear load correspond- ing to the last load step before reaching the maximum shear load was recorded (this last value was difficult to record, unless it was eventually equal to the last load step).

\section{Results of shear tests}

\subsection{Specimens of blocks of aerated autoclaved concrete ( $G x$, $H x$ series and additional specimen Hxrl)}

In the shear test of specimens Gx1, Gx2, Gx3 and Gx4, the applied constant normal stresses were, respectively, $0.50,0.75,1.00$ and $1.25 \mathrm{MPa}$ (Table 3). The relative peak shear stresses were, respectively, around 1.14, 1.54, 1.40 and $1.33 \mathrm{MPa}$ (Table 3 - see results of Gx1 test example in Fig. 2), while the corresponding average peak normal displacements were, respectively, around, 1.7, 0.3, 0.1, $0 \mathrm{~mm}$; and the average peak shear displacements were, respectively, around $-2.5,-0.5,-0.3,-0.1 \mathrm{~mm}$ (Table 3 , Fig. 3); the average residual shear displacements varied between -1.0 and $-2.9 \mathrm{~mm}$.

Table 3

Summary of shear test results series Gx, Hx and additional specimen Hxr1

\begin{tabular}{|c|c|c|c|c|c|c|c|c|c|c|c|c|c|}
\hline \multirow[t]{4}{*}{ Series } & \multicolumn{8}{|c|}{ Test results } & \multicolumn{5}{|c|}{$\begin{array}{l}\text { Normal stress, shear stress, cohesion, friction coefficient } \\
\text { (maximum and residual value) }^{\mathrm{a}}\end{array}$} \\
\hline & \multicolumn{6}{|c|}{$\begin{array}{l}\text { Peak }{ }^{\mathrm{b}} \text { and residual normal and shear } \\
\text { displacement }\left(\times 10^{-2} \mathrm{~mm}\right)\end{array}$} & \multicolumn{2}{|c|}{$\begin{array}{l}\text { Mean peak, } \\
\text { residual } \\
\text { displacement }^{\mathrm{b}}- \\
\text { normal and } \\
\text { shear } \\
\left(\times 10^{-2} \mathrm{~mm}\right)\end{array}$} & \multirow{3}{*}{$\begin{array}{l}\text { Normal } \\
\text { stress } \sigma_{\mathrm{n}} \\
\text { MPa (bar) }\end{array}$} & \multirow{3}{*}{$\begin{array}{l}\text { Maximum lateral } \\
\text { stress } \tau_{\max } \mathrm{MPa} \\
\text { failure (mode) }\end{array}$} & \multirow{3}{*}{$\begin{array}{l}\text { Lateral residual } \\
\text { stress } \tau_{\text {res }} \mathrm{MPa} \\
\text { failure }(\mathrm{desc})\end{array}$} & \multirow[t]{3}{*}{$\begin{array}{l}\text { Cohesion } \tau_{\mathrm{o} \text {,max }} \\
\left(\tau_{\mathrm{o}, \mathrm{res}}\right) \mathrm{MPa}\end{array}$} & \multirow{3}{*}{$\begin{array}{l}\text { Friction } \\
\text { coefficient } \\
\tan \varphi_{\max } \\
\left(\tan \varphi_{\text {res }}\right)^{\mathrm{c}}\end{array}$} \\
\hline & \multicolumn{4}{|c|}{ Normal } & \multicolumn{2}{|l|}{ Shear } & \multirow{2}{*}{$\begin{array}{l}\text { Normal } \\
\varepsilon_{\mathrm{Vm}}\end{array}$} & \multirow{2}{*}{$\frac{\text { Shear }}{\varepsilon_{\mathrm{Tm}}}$} & & & & & \\
\hline & $\varepsilon_{\mathrm{V} 1}$ & $\varepsilon_{\mathrm{V} 2}$ & $\varepsilon_{\mathrm{V} 3}$ & $\varepsilon_{\mathrm{V} 4}$ & $\varepsilon_{\mathrm{T} 5}$ & $\varepsilon_{\mathrm{T} 6}$ & & & & & & & \\
\hline Gx1 & $\begin{array}{l}-80 \\
-76\end{array}$ & $\begin{array}{r}460 \\
-391\end{array}$ & $\begin{array}{l}-130 \\
-176\end{array}$ & $\begin{array}{l}415 \\
282\end{array}$ & $\begin{array}{l}-257 \\
-306\end{array}$ & $\begin{array}{l}-233 \\
-271\end{array}$ & $\begin{array}{r}166 \\
-90\end{array}$ & $\begin{array}{l}-245 \\
-289\end{array}$ & $\begin{array}{r}0.50 \\
(12.5)\end{array}$ & $\begin{array}{l}1.14 \\
\text { (Shear) }\end{array}$ & $\begin{array}{l}0.58 \\
\text { (1a) }\end{array}$ & $\begin{array}{c}0.17 \\
(0.45)\end{array}$ & $\begin{array}{c}1.21 \\
(0.37)\end{array}$ \\
\hline $\mathrm{Gx} 2$ & $\begin{array}{l}-21 \\
-70\end{array}$ & $\begin{array}{l}115 \\
146\end{array}$ & $\begin{array}{r}-53 \\
-165\end{array}$ & $\begin{array}{l}81 \\
47\end{array}$ & $\begin{array}{r}-77 \\
-186\end{array}$ & $\begin{array}{l}-20 \\
-15\end{array}$ & $\begin{array}{r}31 \\
-105\end{array}$ & $\begin{array}{r}-49 \\
-100\end{array}$ & $\begin{array}{r}0.75 \\
(18.2)\end{array}$ & $\begin{array}{l}1.54 \\
\text { (Shear) }\end{array}$ & $\begin{array}{l}0.84 \\
\text { (1a) }\end{array}$ & & \\
\hline Gx3 & $\begin{array}{l}-10 \\
-17\end{array}$ & $\begin{array}{l}31 \\
26\end{array}$ & $\begin{array}{l}-11 \\
-21\end{array}$ & $\begin{array}{l}33 \\
25\end{array}$ & $\begin{array}{r}-35 \\
-272\end{array}$ & $\begin{array}{r}-29 \\
-135\end{array}$ & $\begin{array}{r}11 \\
3\end{array}$ & $\begin{array}{r}-32 \\
-204\end{array}$ & $\begin{array}{r}1.00 \\
(23.9)\end{array}$ & $\begin{array}{l}1.40 \\
\text { (Shear) }\end{array}$ & $\begin{array}{l}0.74 \\
\text { (1a) }\end{array}$ & & \\
\hline $\mathrm{Gx} 4$ & $\begin{array}{l}-12 \\
-22\end{array}$ & $\begin{array}{l}13 \\
26\end{array}$ & $\begin{array}{l}-13 \\
-41\end{array}$ & $\begin{array}{r}13 \\
9\end{array}$ & $\begin{array}{r}1 \\
-48\end{array}$ & $\begin{array}{r}-19 \\
-180\end{array}$ & $\begin{array}{r}0 \\
-7\end{array}$ & $\begin{array}{r}-9 \\
-114\end{array}$ & $\begin{array}{r}1.25 \\
(29.6)\end{array}$ & $\begin{array}{l}1.33 \\
\text { (Shear) }\end{array}$ & $\begin{array}{l}0.92 \\
\text { (1b) }\end{array}$ & & \\
\hline $\mathrm{Hx} 1$ & $\begin{array}{l}-38 \\
-84\end{array}$ & $\begin{array}{l}83 \\
71\end{array}$ & $\begin{array}{r}-42 \\
-109\end{array}$ & $\begin{array}{l}83 \\
45\end{array}$ & $\begin{array}{r}-86 \\
-125\end{array}$ & $\begin{array}{r}-72 \\
-360\end{array}$ & $\begin{array}{r}22 \\
-19\end{array}$ & $\begin{array}{r}-79 \\
-242\end{array}$ & $\begin{array}{r}0.75 \\
(18.2)\end{array}$ & $\begin{array}{l}1.55 \\
\text { (Shear) }\end{array}$ & $\begin{array}{l}0.75 \\
\text { (1a) }\end{array}$ & $\begin{array}{c}0.92 \\
(0.43)\end{array}$ & $\begin{array}{c}0.89 \\
(0.47)\end{array}$ \\
\hline $\mathrm{Hx} 2$ & $\begin{array}{l}-16 \\
-58\end{array}$ & $\begin{array}{l}59 \\
20\end{array}$ & $\begin{array}{l}-31 \\
-67\end{array}$ & $\begin{array}{l}55 \\
18\end{array}$ & $\begin{array}{r}-78 \\
-283\end{array}$ & $\begin{array}{r}-67 \\
-241\end{array}$ & $\begin{array}{r}17 \\
-22\end{array}$ & $\begin{array}{r}-73 \\
-262\end{array}$ & $\begin{array}{r}1.00 \\
(23.9)\end{array}$ & $\begin{array}{l}1.89 \\
\text { (Shear/tension) }\end{array}$ & $\begin{array}{l}0.93 \\
(1 \mathrm{c})\end{array}$ & & \\
\hline $\mathrm{Hx} 3$ & $\begin{array}{r}-25 \\
-108\end{array}$ & $\begin{array}{l}56 \\
22\end{array}$ & $\begin{array}{r}-30 \\
-165\end{array}$ & $\begin{array}{r}60 \\
-32\end{array}$ & $\begin{array}{r}-88 \\
-427\end{array}$ & $\begin{array}{r}-69 \\
-460\end{array}$ & $\begin{array}{r}15 \\
-71\end{array}$ & $\begin{array}{r}-79 \\
-444\end{array}$ & $\begin{array}{r}1.25 \\
(29.6)\end{array}$ & $\begin{array}{l}2.00 \\
\text { (Tension) }\end{array}$ & $\begin{array}{l}0.99 \\
(2 \mathrm{a})\end{array}$ & & \\
\hline Hxrl & $\begin{array}{l}-14 \\
-53\end{array}$ & $\begin{array}{r}81 \\
142\end{array}$ & $\begin{array}{r}-42 \\
-114\end{array}$ & $\begin{array}{l}58 \\
93\end{array}$ & $\begin{array}{r}-44 \\
-324\end{array}$ & $\begin{array}{r}-42 \\
-327\end{array}$ & $\begin{array}{r}20 \\
-9\end{array}$ & $\begin{array}{r}-43 \\
-326\end{array}$ & $\begin{array}{r}0.75 \\
(18.2)\end{array}$ & $\begin{array}{l}1.55 \\
\text { (Shear) }\end{array}$ & $\begin{array}{l}0.86 \\
(1 \mathrm{a})\end{array}$ & & \\
\hline
\end{tabular}

${ }^{\mathrm{a}}$ The peak and residual values of cohesion and friction coefficient $(\tan \varphi)$ were determined from a regression analysis of the failure envelope curve of each series; in the calculation of peak and residual of cohesion for $\mathrm{Gz}$ and $\mathrm{Hz}$ series, it was not possible to obtain reliable results, and zero values could be assumed on safe side.

${ }^{\mathrm{b}}$ Due to the impossibility, in some cases, of recording the peak deformation, this was defined as the value correspondent to the immediately prior load step (the peak deformation values as well as the residuals values were fixed by approximation). The normal and shear deformations are negative when are processed in the sense of the application of the load, respectively, in normal and horizontal direction.

${ }^{c}$ Dimensions (in mm) of the specimens, length, height (height = height ${ }_{\text {block }}+$ height $_{\text {mortar }}$ ), width: Gx, Hx, Bk series $-100 \times 103 \times 100 \mathrm{~mm}$; Gy, Hy, Gz, $\mathrm{Hz}, \mathrm{Bw}, \mathrm{Bv}$ series $-100 \times 105 \times 100 \mathrm{~mm}$ (see also Tables 4-6); Gz, Hz series $-100 \times 105 \times 65 \mathrm{~mm}$ (see also Table 6). 

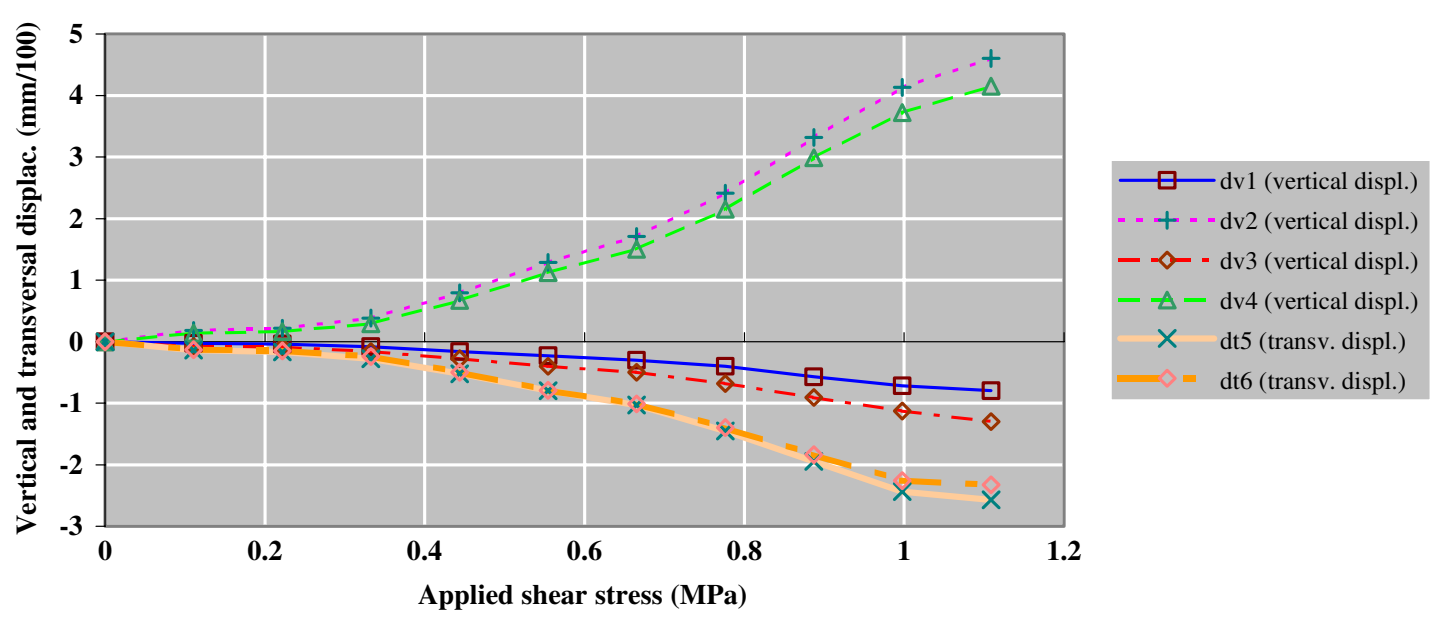

Fig. 2. Normal and shear displacements of specimen Gx1 during the shear test, till peak values were obtained (normal constant stress $\sigma_{\mathrm{n}}=0.50 \mathrm{MPa}-$ cellular autoclaved concrete specimen with simple horizontal joint).

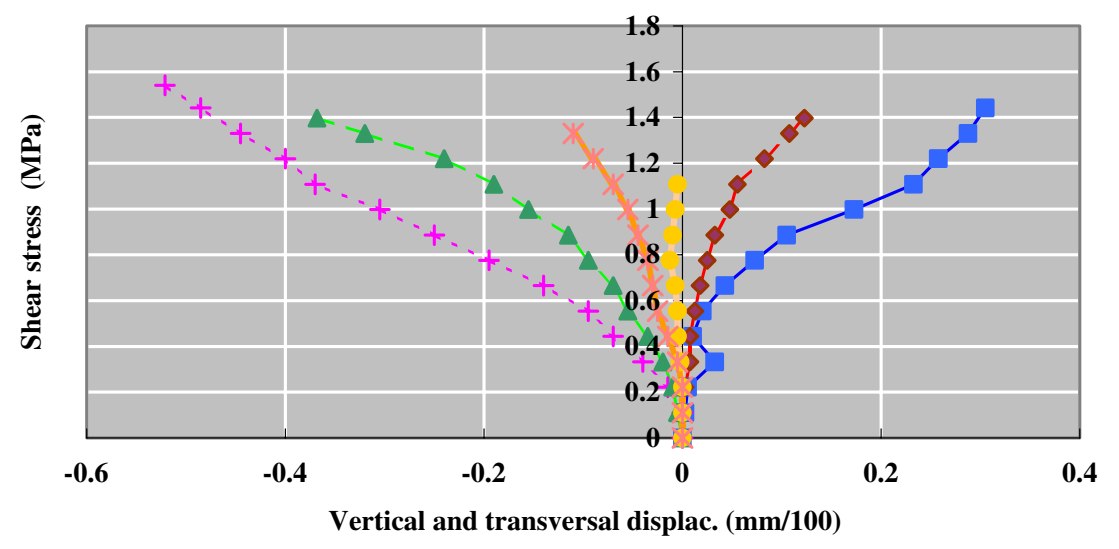

\begin{tabular}{|c|c|}
\hline $\begin{array}{l}-\mathrm{Gx} 2 \text { (ver. displ. - } 0.75 \mathrm{MPa} \text { ) } \\
-\mathrm{Gx} 3 \text { (tran. displ -1.00 MPa) }\end{array}$ & - - + - - Gx2 (tran. displ - 0.75 MPa.) $-\prec-$ - Gx3 (ver. displ. - 1.00 MPa) \\
\hline
\end{tabular}

Fig. 3. Average normal and shear displacements of specimens Gx2, Gx3 and Gx4, during the shear test, till peak values were obtained (normal constant stress $\sigma_{\mathrm{n}}=0.75 ; 1.00 ; 1.25 \mathrm{MPa}$ - lightweight concrete with expanded clay aggregates specimen with simple horizontal joint).

In the shear test of specimens $\mathrm{Hx} 1, \mathrm{Hx} 2$ and $\mathrm{Hx} 3$, the applied constant normal stresses were, respectively, $0.75,1.00$ and $1.25 \mathrm{MPa}$, the relative peak shear stresses were, respectively, around $1.55,1.89$, and $2.00 \mathrm{MPa}$ (Table 3). The mode of failure of specimens in Gx and $\mathrm{Hx}$ series was by slipping along the bed joint of the specimens.

In the additional shear test made on the specimen Hxr1, with the horizontal joint similar to that of $\mathrm{Gx}$ but additionally including a metal expanded meshwork placed in the middle of the thickness of that joint, the constant normal stress applied was approximately $0.75 \mathrm{MPa}$, the relative peak shear stress was $1.54 \mathrm{MPa}$ (the same value of Gx2), the corresponding average peak normal displacements was near $0.2 \mathrm{~mm}$, the peak shear displacement was near $-0.4 \mathrm{~mm}$, and the average residual shear displacement was around $-3.3 \mathrm{~mm}$. Both these two shear displacements values were inferior to the correspondent values of specimen Gx2 (with the same normal applied stress as Hxr1).

\section{Description of the type of failure}

\section{Type of failure 1 :}

(1a) global slipping along the joint;

(1b) slipping along the bed joint with inclined cracking near the lateral face opposite to that of application of shear load.

(1c) slipping and cracking irregular along the joint ("teeth" configuration of cracking);

(1d) local slipping in the bed joint associated with high values of shear deformations and with crushing and grinding of mortar material to inclined cracking beginning from the face of application of shear load and progressing to the base of the specimen. 
Table 4

Summary of shear test results series Gy, Hy, and additional specimen Hyrl

\begin{tabular}{|c|c|c|c|c|c|c|c|c|c|c|c|c|c|}
\hline \multirow[t]{4}{*}{ Series } & \multicolumn{8}{|c|}{ Test results } & \multicolumn{5}{|c|}{$\begin{array}{l}\text { Normal stress, shear stress, cohesion, friction coefficient } \\
\text { (maximum and residual value })^{\mathrm{a}}\end{array}$} \\
\hline & \multicolumn{6}{|c|}{$\begin{array}{l}\text { Peak }{ }^{\mathrm{b}} \text { and residual normal and shear } \\
\text { displacement }\left(\times 10^{-2} \mathrm{~mm}\right)\end{array}$} & \multicolumn{2}{|c|}{$\begin{array}{l}\text { Mean peak, } \\
\text { residual } \\
\text { displacement }^{\mathrm{b}}- \\
\text { normal and } \\
\text { shear } \\
\left(\times 10^{-2} \mathrm{~mm}\right)\end{array}$} & \multirow{3}{*}{$\begin{array}{l}\text { Normal } \\
\text { stress } \sigma_{\mathrm{n}} \\
\text { MPa (bar) }\end{array}$} & \multirow{3}{*}{$\begin{array}{l}\text { Maximum lateral } \\
\text { stress } \tau_{\max } \mathrm{MPa} \\
\text { failure }(\text { desc })\end{array}$} & \multirow{3}{*}{$\begin{array}{l}\text { Lateral residual } \\
\text { stress } \tau_{\text {res }} \mathrm{MPa} \\
\text { failure (mode) }\end{array}$} & \multirow{3}{*}{$\begin{array}{l}\text { Cohesion } \\
\tau_{\mathrm{o}, \max }\left(\tau_{\mathrm{o}, \text { res }}\right) \\
\mathrm{MPa}\end{array}$} & \multirow{3}{*}{$\begin{array}{l}\text { Friction } \\
\text { coeficien } \\
\tan \varphi_{\text {max }} \\
\left(\tan \varphi_{\text {res }}\right)\end{array}$} \\
\hline & \multicolumn{4}{|c|}{ Normal } & \multicolumn{2}{|l|}{ Shear } & \multirow{2}{*}{$\frac{\text { Normal }}{\varepsilon_{\mathrm{Vm}}}$} & \multirow{2}{*}{$\frac{\text { Shear }}{\varepsilon_{\mathrm{Tm}}}$} & & & & & \\
\hline & $\varepsilon_{\mathrm{V} 1}$ & $\varepsilon_{\mathrm{V} 2}$ & $\varepsilon_{\mathrm{V} 3}$ & $\varepsilon_{\mathrm{V} 4}$ & $\varepsilon_{\mathrm{T} 5}$ & $\varepsilon_{\mathrm{T} 6}$ & & & & & & & \\
\hline Gy1 & $\begin{array}{l}-19 \\
-20\end{array}$ & $\begin{array}{l}57 \\
63\end{array}$ & $\begin{array}{l}-40 \\
-46\end{array}$ & $\begin{array}{l}56 \\
34\end{array}$ & $\begin{array}{r}-36 \\
-925\end{array}$ & $\begin{array}{r}-29 \\
-320\end{array}$ & $\begin{array}{r}14 \\
8\end{array}$ & $\begin{array}{r}-33 \\
-623\end{array}$ & $\begin{array}{r}0.75 \\
(18.2)\end{array}$ & $\begin{array}{l}1.11 \\
\text { (Shear) }\end{array}$ & $\begin{array}{l}0.64 \\
(1 d)\end{array}$ & $\begin{array}{c}0.47 \\
(0.20)\end{array}$ & $\begin{array}{c}0.75 \\
(0.72)\end{array}$ \\
\hline Gy2 & $\begin{array}{r}-18 \\
-272\end{array}$ & $\begin{array}{r}44 \\
354\end{array}$ & $\begin{array}{r}-29 \\
-351\end{array}$ & $\begin{array}{r}42 \\
285\end{array}$ & $\begin{array}{l}-104 \\
-571\end{array}$ & $\begin{array}{l}-100 \\
-327\end{array}$ & $\begin{array}{r}10 \\
4\end{array}$ & $\begin{array}{l}-102 \\
-449\end{array}$ & $\begin{array}{r}1.00 \\
(23.9)\end{array}$ & $\begin{array}{l}1.13 \\
\text { (Shear) }\end{array}$ & $\begin{array}{l}1.11 \\
(1 d)\end{array}$ & & \\
\hline Gy3 & $\begin{array}{r}-19 \\
-127\end{array}$ & $\begin{array}{r}48 \\
132\end{array}$ & $\begin{array}{r}-27 \\
-126\end{array}$ & $\begin{array}{r}63 \\
142\end{array}$ & $\begin{array}{l}-155 \\
-986\end{array}$ & $\begin{array}{l}-194 \\
-412\end{array}$ & $\begin{array}{r}16 \\
6\end{array}$ & $\begin{array}{l}-175 \\
-699\end{array}$ & $\begin{array}{r}1.25 \\
(29.6)\end{array}$ & $\begin{array}{l}1.40 \\
\text { (Shear) }\end{array}$ & $\begin{array}{l}1.02 \\
\text { (1d) }\end{array}$ & & \\
\hline Gy4 & $\begin{array}{l}-38 \\
-47\end{array}$ & $\begin{array}{l}65 \\
62\end{array}$ & $\begin{array}{l}-65 \\
-80\end{array}$ & $\begin{array}{l}63 \\
44\end{array}$ & $\begin{array}{r}-97 \\
-565\end{array}$ & $\begin{array}{r}-92 \\
-196\end{array}$ & $\begin{array}{r}6 \\
-5\end{array}$ & $\begin{array}{r}-95 \\
-381\end{array}$ & $\begin{array}{r}2.00 \\
(46.7)\end{array}$ & $\begin{array}{l}2.00 \\
\text { (Shear) }\end{array}$ & $\begin{array}{l}1.64 \\
(1 d)\end{array}$ & & \\
\hline Hy1 & $\begin{array}{r}-6 \\
9\end{array}$ & $\begin{array}{l}12 \\
35\end{array}$ & $\begin{array}{r}-14 \\
-9\end{array}$ & $\begin{array}{r}6 \\
17\end{array}$ & $\begin{array}{r}-34 \\
-464\end{array}$ & $\begin{array}{l}-25 \\
-74\end{array}$ & $\begin{array}{r}-1 \\
13\end{array}$ & $\begin{array}{r}-30 \\
-269\end{array}$ & $\begin{array}{r}0.75 \\
(18.2)\end{array}$ & $\begin{array}{l}0.88 \\
\text { (Shear) }\end{array}$ & $\begin{array}{l}0.74 \\
(1 d)\end{array}$ & $\begin{array}{c}0.20 \\
(0.32)\end{array}$ & $\begin{array}{c}0.99 \\
(0.74)\end{array}$ \\
\hline Hy2 & $\begin{array}{r}1 \\
-33\end{array}$ & $\begin{array}{r}24 \\
109\end{array}$ & $\begin{array}{r}-2 \\
-76\end{array}$ & $\begin{array}{l}11 \\
65\end{array}$ & $\begin{array}{r}-52 \\
-595\end{array}$ & $\begin{array}{r}-41 \\
-321\end{array}$ & $\begin{array}{l}11 \\
16\end{array}$ & $\begin{array}{r}-47 \\
-458\end{array}$ & $\begin{array}{r}1.00 \\
(23.9)\end{array}$ & $\begin{array}{l}1.11 \\
\text { (Shear) }\end{array}$ & $\begin{array}{l}0.98 \\
\text { (1d) }\end{array}$ & & \\
\hline Hy3 & $\begin{array}{l}-255 \\
-310\end{array}$ & $\begin{array}{l}295 \\
352\end{array}$ & $\begin{array}{l}-247 \\
-289\end{array}$ & $\begin{array}{l}257 \\
317\end{array}$ & $\begin{array}{l}-880 \\
-970\end{array}$ & $\begin{array}{l}-251 \\
-321\end{array}$ & $\begin{array}{l}13 \\
18\end{array}$ & $\begin{array}{l}-566 \\
-646\end{array}$ & $\begin{array}{r}1.25 \\
(29.6)\end{array}$ & $\begin{array}{l}1.66 \\
\text { (Shear) }\end{array}$ & $\begin{array}{l}1.59 \\
(1 d)\end{array}$ & & \\
\hline Hy4 & $\begin{array}{r}-16 \\
-109\end{array}$ & $\begin{array}{r}27 \\
100\end{array}$ & $\begin{array}{r}-17 \\
-139\end{array}$ & $\begin{array}{l}35 \\
87\end{array}$ & $\begin{array}{r}-140 \\
510\end{array}$ & $\begin{array}{l}-134 \\
-170\end{array}$ & $\begin{array}{r}7 \\
-15\end{array}$ & $\begin{array}{r}-137 \\
170\end{array}$ & $\begin{array}{r}2.00 \\
(46.7)\end{array}$ & $\begin{array}{l}2.11 \\
\text { (Shear) }\end{array}$ & $\begin{array}{l}1.68 \\
(1 d)\end{array}$ & & \\
\hline Hyr1 & $\begin{array}{l}-29 \\
-41\end{array}$ & $\begin{array}{r}61 \\
118\end{array}$ & $\begin{array}{l}-40 \\
-62\end{array}$ & $\begin{array}{r}52 \\
103\end{array}$ & $\begin{array}{l}-153 \\
-403\end{array}$ & $\begin{array}{l}-149 \\
-431\end{array}$ & $\begin{array}{l}11 \\
30\end{array}$ & $\begin{array}{l}-151 \\
-417\end{array}$ & $\begin{array}{r}0.75 \\
(18.2)\end{array}$ & $\begin{array}{l}1.43 \\
\text { (Shear) }\end{array}$ & $\begin{array}{l}0.88 \\
(1 \mathrm{~d})\end{array}$ & & \\
\hline
\end{tabular}

${ }^{a}$ The peak and residual values of cohesion and friction coefficient $(\tan \varphi)$ were determined from a regression analysis of the failure envelope curve of each series; in the calculation of peak and residual of cohesion for $\mathrm{Gz}$ and $\mathrm{Hz}$ series, it was not possible to obtain reliable results, and zero values could be assumed on safe side.

${ }^{\mathrm{b}}$ Due to the impossibility, in some cases, of recording the peak deformation, this was defined as the value correspondent to the immediately prior load step (the peak deformation values as well as the residuals values were fixed by approximation). The normal and shear deformations are negative when are processed in the sense of the application of the load, respectively, in normal and horizontal direction.
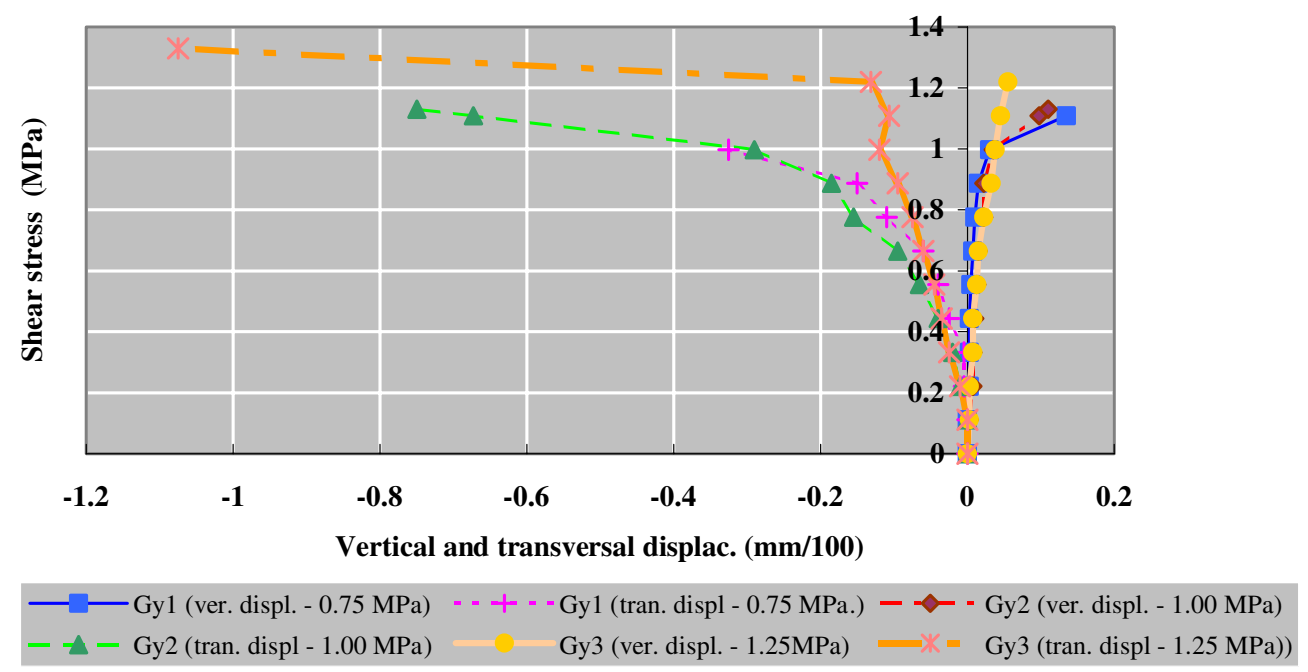

Fig. 4. Average normal and shear displacements of specimens Gy1, Gy2 and Gy3, during the shear test, till peak values were obtained (normal constant stress $\sigma_{\mathrm{n}}=0.75 ; 1.00 ; 1.25 \mathrm{MPa}$ - cellular autoclaved concrete specimen with simple horizontal joint). 


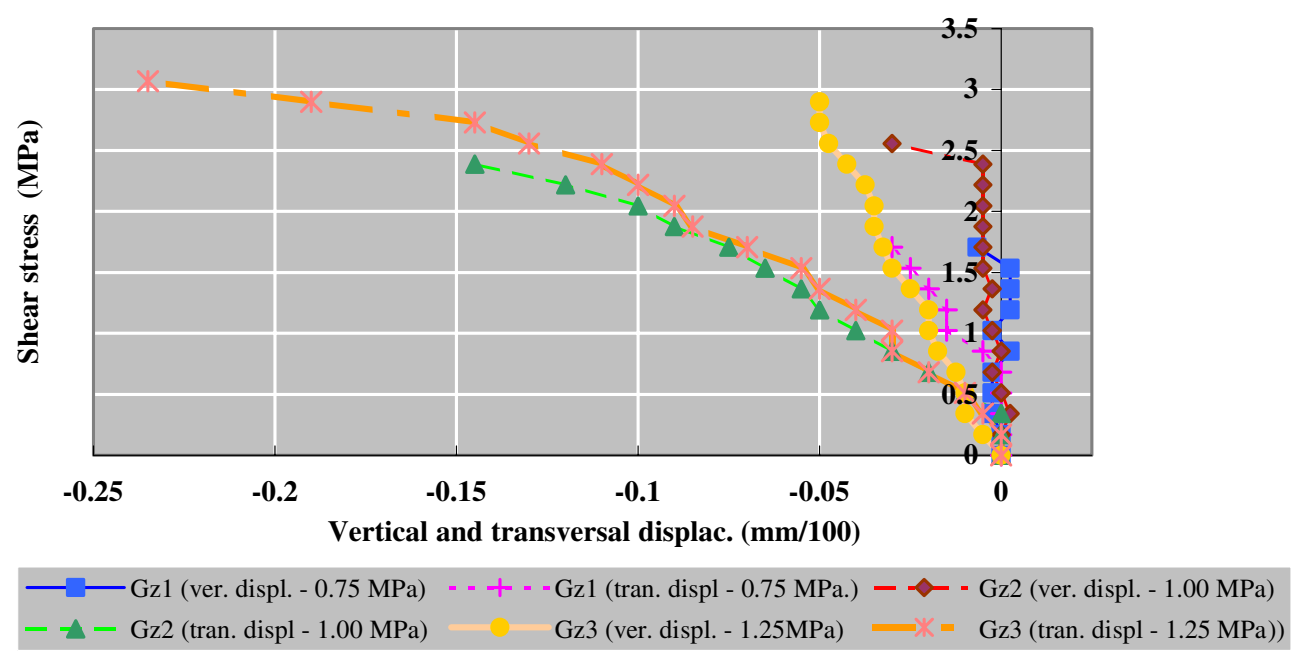

Fig. 5. Average normal and shear displacements of specimens Gz1, Gz2 and Gz3, during the shear test, till peak values were obtained (normal constant stress $\sigma_{\mathrm{n}}=0.75 ; 1.00 ; 1.25 \mathrm{MPa}$ - clay solid specimen with simple horizontal joint).

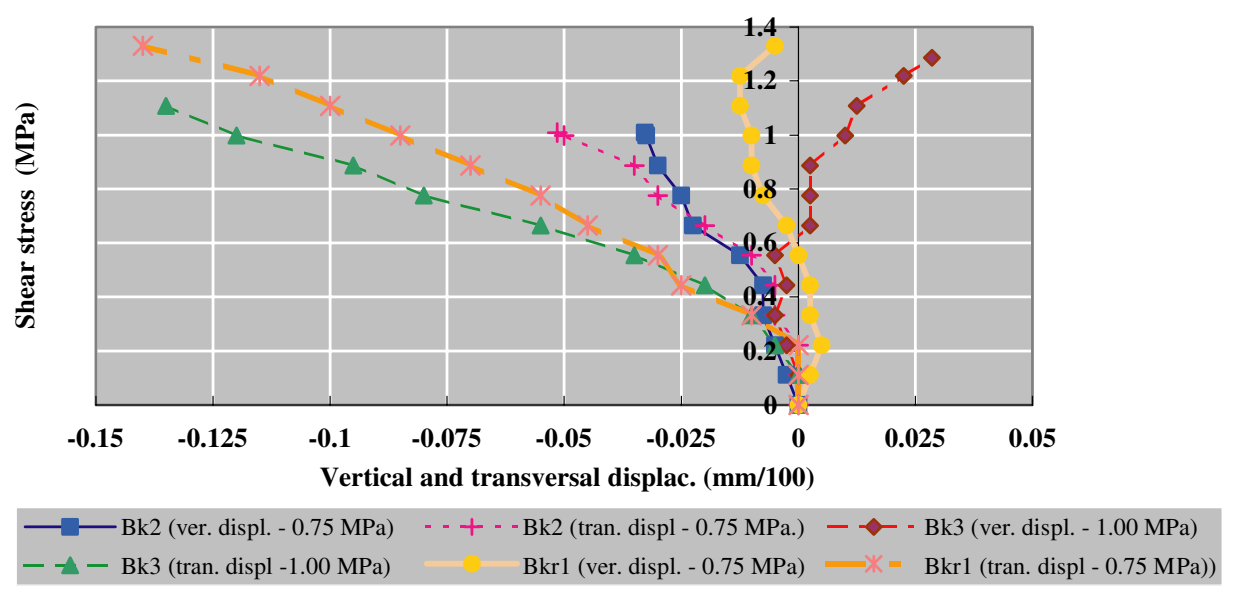

Fig. 6. Average normal and shear displacements of specimens Bk2, Bk3 and Bkr1, during the shear test, till peak values were obtained (normal constant stress $\sigma_{\mathrm{n}}=0.75 ; 1.00 ; 0.75 \mathrm{MPa}-$ cellular autoclaved concrete/concrete specimen).

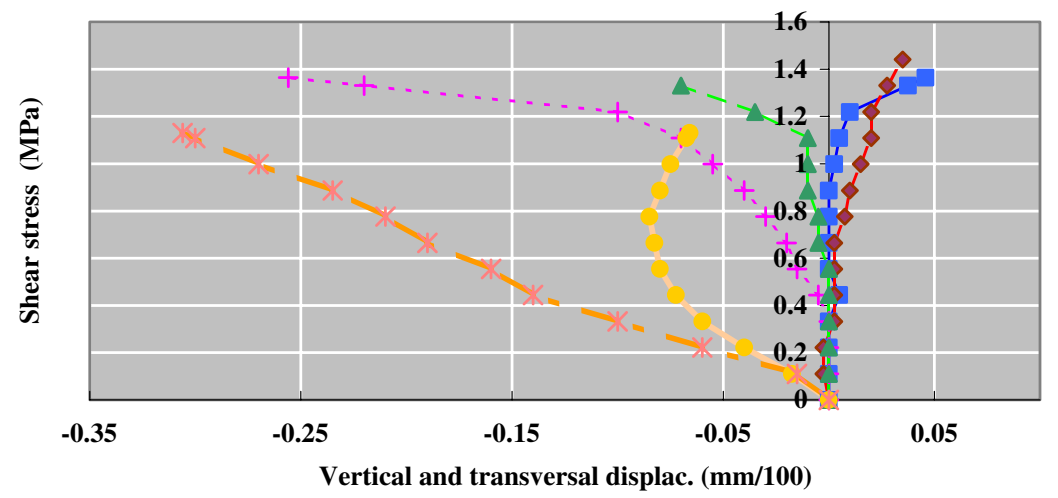

$\begin{array}{rrr}- \text { Bw1 (ver. displ. - 0.75 MPa) } & -+-- \text { Bw1 (tran. displ - 0.75 MPa.) } & -\leftarrow-\text { Bw2 (ver. displ. }-1.00 \mathrm{MPa}) \\ -\triangle-\text { Bw2 (tran. displ -1.00 MPa) } & \text { Bwr1 (ver. displ. - 0.75 MPa) } & \text { Bwr1 (tran. displ - 0.75 MPa) })\end{array}$

Fig. 7. Average normal and shear displacements of specimens Bw1, Bw2 and Bwr1, during the shear test, till peak values were obtained (normal constant stress $\sigma_{\mathrm{n}}=0.75 ; 1.00 ; 0.75 \mathrm{MPa}-$ lightweight concrete/concrete specimen). 
Type of failure 2:

(2a) inclined cracking beginning from the face of application of shear load and progressing to the base of the specimen and associated to a light cracking near the lateral face opposite to that of application of shear load.

\subsection{Specimens of blocks of lightweight concrete with expanded clay aggregates (Gy, Hy series and additional specimen Hyrl)}

In the G-type shear test specimens, the applied constant normal stresses varied between 0.75 and $2.00 \mathrm{MPa}$ (Table 4). The relative peak shear stresses were, around 1.11, 1.13, 1.40 and $2.00 \mathrm{MPa}$ (Fig. 4).

In the H-type shear test specimens, the constant normal stress applied varied between 0.75 and $2.00 \mathrm{MPa}$. The relative peak shear stresses were, respectively, around 0.88, $1.11,1.66$ and $2.11 \mathrm{MPa}$ (see Table 4).

The mode of failure of specimens, in Gy and Hy series, was, generally, by slipping along the bed joint of the specimens, and for Hy specimens, a separation along the vertical joint was detected for a load near that of the relative peak shear stress, as in the Hx series. The shear test on the additional specimen Hyr1, with the horizontal bed joint similar to that of Gy but additionally including a expanded metal meshwork (like Hxr1), and where the constant normal stress applied was $0.75 \mathrm{MPa}$, the relative peak shear stress was $1.43 \mathrm{MPa}$ (higher than Gyl that was near $1.11 \mathrm{MPa}$ and it had the same normal applied stress as Hyr1), the corresponding average peak normal displacement was close to that of Gyl but the average peak shear displacements was lesser than that observed in these last specimen.

\subsection{Specimens of solid clay bricks ( $\mathrm{Gz}, \mathrm{Hz}$ series)}

In the shear test of specimens Gz1, Gz2, Gz3, the applied constant normal stresses were, respectively, 0.75, 1.00, 1.25 MPa (see Table 5, Fig. 5).

In the shear test of specimens $\mathrm{Hz} 1, \mathrm{~Hz} 2$, and $\mathrm{Hz} 3$, the applied constant normal stresses were, respectively, $0.75,1.00$, 1.25 MPa (Table 5). The mode of failure of specimens, in Gz and Hzy series, was similar to that of Gy and Hy (by slipping along the bed joint).

\subsection{Mixed specimens (Bk, Bw and Bv series)}

In the shear test of specimens $\mathrm{Bk} 1, \mathrm{Bk} 2, \mathrm{Bk} 3, \mathrm{Bk} 4$ the applied constant normal stresses were, respectively, 0.50, $0.75,1.00$ and $1.25 \mathrm{MPa}$ (see Table 6, Fig. 6). In the shear test of specimens $\mathrm{Bw} 1, \mathrm{Bw} 2$, and $\mathrm{Bw} 3$, the applied con-

Table 5

Summary of shear test results series $\mathrm{Gz}, \mathrm{Hz}$

\begin{tabular}{|c|c|c|c|c|c|c|c|c|c|c|c|c|c|}
\hline \multirow[t]{4}{*}{ Series } & \multicolumn{8}{|c|}{ Test results } & \multicolumn{5}{|c|}{$\begin{array}{l}\text { Normal stress, shear stress, cohesion, friction coefficient (maximum and } \\
\text { residual value) }\end{array}$} \\
\hline & \multicolumn{6}{|c|}{$\begin{array}{l}\text { Peak }{ }^{\mathrm{b}} \text { and residual normal and shear } \\
\text { displacement }\left(\times 10^{-2} \mathrm{~mm}\right)\end{array}$} & \multicolumn{2}{|c|}{$\begin{array}{l}\text { Mean peak, } \\
\text { residual } \\
\text { displacement }^{\mathrm{b}}- \\
\text { normal and } \\
\text { shear } \\
\left(\times 10^{-2} \mathrm{~mm}\right)\end{array}$} & $\begin{array}{l}\text { Normal stress } \\
\sigma_{\mathrm{n}} \mathrm{MPa} \text { (bar) }\end{array}$ & $\begin{array}{l}\text { Maximum } \\
\text { lateral stress }\end{array}$ & $\begin{array}{l}\text { Lateral } \\
\text { residual }\end{array}$ & $\begin{array}{l}\text { Cohesion } \\
\tau_{\mathrm{o}, \max }\left(\tau_{\mathrm{o}, \mathrm{res}}\right)\end{array}$ & $\begin{array}{l}\text { Friction } \\
\text { coeficient }\end{array}$ \\
\hline & \multicolumn{4}{|c|}{ Normal } & \multicolumn{2}{|l|}{ Shear } & \multirow{2}{*}{$\frac{\text { Normal }}{\varepsilon_{\mathrm{Vm}}}$} & Shear & & & & & \\
\hline & $\varepsilon_{\mathrm{V} 1}$ & $\varepsilon_{\mathrm{V} 2}$ & $\varepsilon_{\mathrm{V} 3}$ & $\varepsilon_{\mathrm{V} 4}$ & $\varepsilon_{\mathrm{T} 5}$ & $\varepsilon_{\mathrm{T} 6}$ & & $\varepsilon_{\mathrm{Tm}}$ & & & & & \\
\hline \multirow[t]{2}{*}{ Gz1 } & -3 & 4 & -3 & 3 & -5 & 0 & 0 & -3 & 0.75 & 1.71 & 1.52 & 0 & 0 \\
\hline & -9 & 10 & -10 & 5 & -86 & -76 & -1 & -81 & (12.2) & (Shear) & (1d) & (0) & (0) \\
\hline \multirow[t]{2}{*}{ Gz2 } & -10 & 10 & -11 & 9 & -15 & -14 & -1 & -15 & 1.00 & 2.56 & 1.59 & & \\
\hline & 18 & 36 & -38 & -21 & -602 & -601 & -1 & -602 & (15.9) & (Shear) & (1d) & & \\
\hline \multirow[t]{2}{*}{ Gz3 } & -13 & 3 & -12 & 2 & -22 & -16 & -5 & -19 & 1.25 & 3.07 & 1.86 & & \\
\hline & -43 & -36 & -36 & -31 & -686 & -224 & -37 & -455 & (19.6) & (Shear) & (1d) & & \\
\hline \multirow[t]{2}{*}{$\mathrm{Hzl}$} & -2 & -1 & -2 & 0 & -15 & -15 & -1 & -15 & 0.75 & 1.84 & 1.65 & 0 & 0 \\
\hline & -9 & 18 & -15 & 13 & -338 & -193 & -2 & -266 & $(12.2)$ & (Shear) & (1d) & (0) & $(0)$ \\
\hline \multirow[t]{2}{*}{$\mathrm{Hz} 2$} & -18 & 12 & -17 & 12 & -56 & -41 & -3 & -49 & 1.00 & 2.52 & 2.03 & & \\
\hline & -146 & 122 & -157 & 107 & -337 & -77 & -19 & -207 & (15.9) & (Shear) & (1d) & & \\
\hline \multirow[t]{2}{*}{$\mathrm{Hz} 3$} & -20 & 19 & -19 & 27 & -134 & -125 & 2 & -130 & 1.25 & 2.98 & 2.51 & & \\
\hline & -105 & -142 & -126 & -127 & 619 & 603 & -125 & 611 & (19.6) & (Shear) & $(1 d)$ & & \\
\hline
\end{tabular}

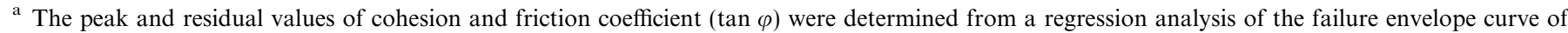

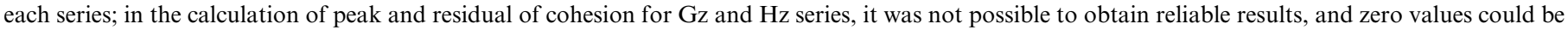
assumed on safe side.

b Due to the impossibility, in some cases, of recording the peak deformation, this was defined as the value correspondent to the immediately prior load

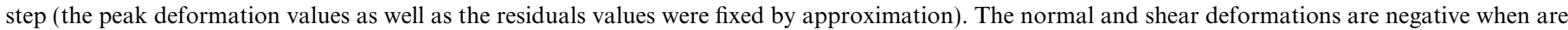
processed in the sense of the application of the load, respectively, in normal and horizontal direction. 
Table 6

Summary of shear test results series Bk, Bw, Bv and additional specimens Bkr1, Bwr1 (mixed specimens)

\begin{tabular}{|c|c|c|c|c|c|c|c|c|c|c|c|c|c|}
\hline \multirow[t]{4}{*}{ Series } & \multicolumn{8}{|c|}{ Test results } & \multicolumn{5}{|c|}{$\begin{array}{l}\text { Normal stress, shear stress, cohesion, friction coefficient (maximum and residual } \\
\text { value) }^{\mathrm{a}}\end{array}$} \\
\hline & \multicolumn{6}{|c|}{$\begin{array}{l}\text { Peak }{ }^{\mathrm{b}} \text { and residual normal and shear } \\
\text { displacement }\left(\times 10^{-2} \mathrm{~mm}\right)\end{array}$} & \multicolumn{2}{|c|}{$\begin{array}{l}\text { Mean peak, } \\
\text { residual } \\
\text { displacement }^{\mathrm{b}}- \\
\text { normal and } \\
\text { shear } \\
\left(\times 10^{-2} \mathrm{~mm}\right)\end{array}$} & \multirow[t]{3}{*}{$\begin{array}{l}\text { Normal stress } \\
\sigma_{\mathrm{n}} \mathrm{MPa} \text { (bar) }\end{array}$} & \multirow{3}{*}{$\begin{array}{l}\text { Maximum } \\
\text { lateral stress } \\
\tau_{\max } \mathrm{MPa} \\
\text { failure (mode) }\end{array}$} & \multirow{3}{*}{$\begin{array}{l}\text { Lateral } \\
\text { residual } \\
\text { stress } \tau_{\text {res }} \\
\text { MPa failure } \\
\text { (desc) }\end{array}$} & \multirow[t]{3}{*}{$\begin{array}{l}\text { Cohesion } \\
\tau_{\mathrm{o}, \max }\left(\tau_{\mathrm{o}, \mathrm{res}}\right) \mathrm{MPa}\end{array}$} & \multirow{3}{*}{$\begin{array}{l}\text { Friction } \\
\text { coefficient } \\
\tan \varphi_{\max } \\
\left(\tan \varphi_{\text {res }}\right)\end{array}$} \\
\hline & \multicolumn{4}{|c|}{$\underline{\text { Normal }}$} & \multicolumn{2}{|l|}{ Shear } & \multirow{2}{*}{$\begin{array}{l}\text { Normal } \\
\varepsilon_{\mathrm{Vm}}\end{array}$} & \multirow{2}{*}{$\frac{\text { Shear }}{\varepsilon_{\mathrm{Tm}}}$} & & & & & \\
\hline & $\varepsilon_{\mathrm{V} 1}$ & $\varepsilon_{\mathrm{V} 2}$ & $\varepsilon_{\mathrm{V} 3}$ & $\varepsilon_{\mathrm{V} 4}$ & $\varepsilon_{\mathrm{T} 5}$ & $\varepsilon_{\mathrm{T} 6}$ & & & & & & & \\
\hline Bk1 & $\begin{array}{r}1 \\
-10\end{array}$ & $\begin{array}{l}30 \\
21\end{array}$ & $\begin{array}{l}-43 \\
-56\end{array}$ & $\begin{array}{l}-12 \\
-26\end{array}$ & $\begin{array}{l}-378 \\
-758\end{array}$ & $\begin{array}{l}-174 \\
-212\end{array}$ & $\begin{array}{r}-6 \\
-18\end{array}$ & $\begin{array}{l}-276 \\
-485\end{array}$ & $\begin{array}{c}0.50 \\
(12.5)\end{array}$ & $\begin{array}{l}0.67 \\
\text { (Shear) }\end{array}$ & $\begin{array}{l}0.39 \\
(1 \mathrm{a})\end{array}$ & $\begin{array}{c}0.64 \\
(0.36)\end{array}$ & $\begin{array}{c}0.72 \\
(0.83)\end{array}$ \\
\hline $\mathrm{Bk} 2$ & $\begin{array}{l}-8 \\
-7\end{array}$ & $\begin{array}{r}2 \\
12\end{array}$ & $\begin{array}{r}-9 \\
-27\end{array}$ & $\begin{array}{r}2 \\
-6\end{array}$ & $\begin{array}{r}-5 \\
-240\end{array}$ & $\begin{array}{r}-5 \\
-228\end{array}$ & $\begin{array}{l}-3 \\
-7\end{array}$ & $\begin{array}{r}-5 \\
-234\end{array}$ & $\begin{array}{r}0.75 \\
(18.2)\end{array}$ & $\begin{array}{l}1.01 \\
\text { (Shear) }\end{array}$ & $\begin{array}{l}0.55 \\
\text { (1a) }\end{array}$ & & \\
\hline $\mathrm{Bk} 3$ & $\begin{array}{r}-6 \\
9\end{array}$ & $\begin{array}{l}15 \\
35\end{array}$ & $\begin{array}{r}-12 \\
37\end{array}$ & $\begin{array}{r}12 \\
-8\end{array}$ & $\begin{array}{r}-22 \\
-364\end{array}$ & $\begin{array}{r}-11 \\
-263\end{array}$ & $\begin{array}{r}2 \\
-3\end{array}$ & $\begin{array}{r}-17 \\
-314\end{array}$ & $\begin{array}{r}1.00 \\
(23.9)\end{array}$ & $\begin{array}{l}1.29 \\
\text { (Shear) }\end{array}$ & $\begin{array}{l}0.78 \\
\text { (1a) }\end{array}$ & & \\
\hline Bk4 & $\begin{array}{l}-15 \\
-19\end{array}$ & $\begin{array}{r}7 \\
25\end{array}$ & $\begin{array}{l}-11 \\
-36\end{array}$ & $\begin{array}{l}11 \\
11\end{array}$ & $\begin{array}{r}-16 \\
-162\end{array}$ & $\begin{array}{r}-9 \\
-123\end{array}$ & $\begin{array}{l}-2 \\
-5\end{array}$ & $\begin{array}{r}-13 \\
-143\end{array}$ & $\begin{array}{r}1.25 \\
(29.6)\end{array}$ & $\begin{array}{l}1.29 \\
\text { (Shear) }\end{array}$ & $\begin{array}{l}0.78 \\
\text { (1a) }\end{array}$ & & \\
\hline Bkr1 & $\begin{array}{l}-14 \\
-10\end{array}$ & $\begin{array}{r}6 \\
16\end{array}$ & $\begin{array}{r}-9 \\
-17\end{array}$ & $\begin{array}{l}15 \\
15\end{array}$ & $\begin{array}{l}-18 \\
-50\end{array}$ & $\begin{array}{r}-10 \\
-327\end{array}$ & $\begin{array}{r}-1 \\
1\end{array}$ & $\begin{array}{r}-14 \\
-189\end{array}$ & $\begin{array}{r}0.75 \\
(18.2)\end{array}$ & $\begin{array}{l}1.44 \\
\text { (Shear) }\end{array}$ & $\begin{array}{l}1.11 \\
\text { (1a) }\end{array}$ & & \\
\hline Bw1 & $\begin{array}{r}-4 \\
-32\end{array}$ & $\begin{array}{l}14 \\
88\end{array}$ & $\begin{array}{r}-7 \\
-25\end{array}$ & $\begin{array}{r}12 \\
9\end{array}$ & $\begin{array}{r}-19 \\
-251\end{array}$ & $\begin{array}{r}-25 \\
-264\end{array}$ & $\begin{array}{l}4 \\
3\end{array}$ & $\begin{array}{r}-22 \\
-258\end{array}$ & $\begin{array}{r}0.75 \\
(18.2)\end{array}$ & $\begin{array}{l}1.36 \\
\text { (Shear) }\end{array}$ & $\begin{array}{l}0.98 \\
(1 \mathrm{~d})\end{array}$ & - & - \\
\hline Bw2 & $\begin{array}{r}-2 \\
3\end{array}$ & $\begin{array}{r}9 \\
24\end{array}$ & $\begin{array}{l}-5 \\
-8\end{array}$ & $\begin{array}{r}9 \\
13\end{array}$ & $\begin{array}{r}-11 \\
-106\end{array}$ & $\begin{array}{r}-3 \\
-72\end{array}$ & $\begin{array}{l}3 \\
8\end{array}$ & $\begin{array}{r}-7 \\
-89\end{array}$ & $\begin{array}{r}1.00 \\
(23.9)\end{array}$ & $\begin{array}{l}1.44 \\
\text { (Shear) }\end{array}$ & $\begin{array}{l}1.08 \\
(1 \mathrm{~d})\end{array}$ & & \\
\hline Bw3 & $\begin{array}{r}-19 \\
1\end{array}$ & $\begin{array}{l}30 \\
58\end{array}$ & $\begin{array}{l}-34 \\
-39\end{array}$ & $\begin{array}{l}12 \\
13\end{array}$ & $\begin{array}{r}-35 \\
-206\end{array}$ & $\begin{array}{r}0 \\
-169\end{array}$ & $\begin{array}{r}-3 \\
8\end{array}$ & $\begin{array}{r}-18 \\
-188\end{array}$ & $\begin{array}{r}1.25 \\
(29.6)\end{array}$ & $\begin{array}{l}1.71 \\
\text { (Shear) }\end{array}$ & $\begin{array}{l}1.43 \\
\text { (1d) }\end{array}$ & & \\
\hline Bwr1 & $\begin{array}{l}-35 \\
-18\end{array}$ & $\begin{array}{l}31 \\
47\end{array}$ & $\begin{array}{l}-44 \\
-44\end{array}$ & $\begin{array}{l}21 \\
18\end{array}$ & $\begin{array}{r}-42 \\
-127\end{array}$ & $\begin{array}{l}-18 \\
-90\end{array}$ & $\begin{array}{r}-7 \\
1\end{array}$ & $\begin{array}{r}-30 \\
-109\end{array}$ & $\begin{array}{r}0.75 \\
(18.2)\end{array}$ & $\begin{array}{l}1.13 \\
\text { (Shear) }\end{array}$ & $\begin{array}{l}0.76 \\
(1 \mathrm{~d})\end{array}$ & & \\
\hline Bv1 & $\begin{array}{r}-4 \\
-104\end{array}$ & $\begin{array}{r}7 \\
207\end{array}$ & $\begin{array}{r}-7 \\
-161\end{array}$ & $\begin{array}{r}6 \\
149\end{array}$ & $\begin{array}{r}-15 \\
-750\end{array}$ & $\begin{array}{r}-7 \\
-713\end{array}$ & $\begin{array}{r}1 \\
23\end{array}$ & $\begin{array}{r}-11 \\
-732\end{array}$ & $\begin{array}{r}0.75 \\
(18.2)\end{array}$ & $\begin{array}{l}1.01 \\
\text { (Shear) }\end{array}$ & $\begin{array}{l}0.95 \\
(1 d)\end{array}$ & & \\
\hline Bv2 & $\begin{array}{l}-13 \\
-\end{array}$ & $\begin{array}{c}21 \\
-\end{array}$ & $\begin{array}{l}-19 \\
-\end{array}$ & $\begin{array}{l}23 \\
-\end{array}$ & $\begin{array}{l}-159 \\
-\end{array}$ & $\begin{array}{l}-118 \\
-\end{array}$ & $\begin{array}{r}3 \\
-\quad\end{array}$ & $\begin{array}{l}-139 \\
-\end{array}$ & $\begin{array}{r}0.75 \\
(18.2)\end{array}$ & (Shear) & $\begin{array}{l}0.98 \\
(1 d)\end{array}$ & & \\
\hline
\end{tabular}

${ }^{a}$ The peak and residual values of cohesion and friction coefficient $(\tan \varphi)$ were determined from a regression analysis of the failure envelope curve of each series; in the calculation of peak and residual of cohesion for $\mathrm{Gz}$ and $\mathrm{Hz}$ series, it was not possible to obtain reliable results, and zero values could be assumed on safe side.

${ }^{\mathrm{b}}$ Due to the impossibility, in some cases, of recording the peak deformation, this was defined as the value correspondent to the immediately prior load step (the peak deformation values as well as the residuals values were fixed by approximation). The normal and shear deformations are negative when are processed in the sense of the application of the load, respectively, in normal and horizontal direction.

stant normal stresses were, respectively, 0.75, 1.00, 1.25 MPa (see Table 6, Fig. 7). In the shear test of specimens Bv1, Bv2 the applied constant normal stress was in both specimens $0.75 \mathrm{MPa}$. The mode of failure of specimens, in $\mathrm{Bk}$ and $\mathrm{Bw}$ series, was generally similar to that of Gy and Hy (by slipping along the bed joint), but in $\mathrm{Bw}$ there were also signs of progressive crushing and grinding of the mortar material around the cracking surface. The shear test on the additional specimens Bkrl and Bwr1, with the horizontal bed joint similar to that of, respectively, $\mathrm{Bk}$ and $\mathrm{Bw}$ but additionally including a expanded metal meshwork in the middle position of the mortar joint thickness (like Hxr1 and Hyr1), and where the constant normal stress applied was $0.75 \mathrm{MPa}$, the relative peak shear stress was, respectively, 1.44 and $1.13 \mathrm{MPa}$.

\subsection{Shear strength, mode II fracture energy, dilatancy angle}

Here, the relation of the shear strength and the applied constant normal load in the shear tests $(\mathrm{Gx}, \mathrm{Hx}, \mathrm{Gy}, \mathrm{Hy}$, $\mathrm{Gz}, \mathrm{Hz}$ series) as well as the corresponding linear best fit was computed, and a tentative interpretation of the results with the relation of Mohr-Coulomb was made

$\tau_{\mathrm{u}}=f_{\mathrm{vko}}+\sigma_{\mathrm{d}} \tan (\varphi)$,

where $f_{\text {vko }}$ is the initial shear strength; $\sigma_{\mathrm{d}}$ is the normal stress in the bed joints; $\tan (\varphi)$ is the tangent of internal friction angle.

The initial shear strength (cohesion), $f_{\text {bvko, obtained }}$ from linear regression of triaxial tests of $\mathrm{Gx}, \mathrm{Hx}, \mathrm{Gy}, \mathrm{Hy}$ were, respectively, around $0.17,0.92,0.47,0.20 \mathrm{MPa}$, but 
these values could not be considered totally reliable due to uncertainties related with the evaluation of post-peak and the influence of the reduced shear failure plane due to locally tensile failure of the specimens. Although these limitations founded in the study, from the calculated results (Fig. 8), it seems possible to consider that the values of peak shear stress (and the associated failure envelope, for the range of normal stress applied in each series) show some dependence on the type of units (material X, Y or $\mathrm{Z}$ ), and mortar used in the joints.

Mode II fracture energy GfII, was approximately evaluated calculating the area between the shear stress-shear displacement and the horizontal line defined by residual shear strength.

Analyzing the results (Fig. 9), GfII apparently show some dependence on the type of specimen material and a tendency, in certain series ( $\mathrm{Hx}$ and $\mathrm{Hy}$ ), for an increase of GfII when normal applied stress is also increased (see Fig. 9).

Dilatancy angle $\psi$ describes the relation between normal displacement and shear displacement, and the ratio between these two variables is $\tan \psi$, which usually assumes positive values. From the results of the shear tests (Fig. 8), dilatancy apparently, in some series (example of Gx and Gy), decreased and tended to zero when normal stress was gradually increased, (as can be seen in Fig. 10) and, probably, it also decreased during the shear test, with increasing shear displacements, due to progressive crushing and grinding of the mortar material around the cracking surface.

\section{Reflections about the results and final considerations}

The results of the shear tests allow to suppose that the inclusion of an additional vertical joint in $\mathrm{Hx}$, $\mathrm{Hy}$ and

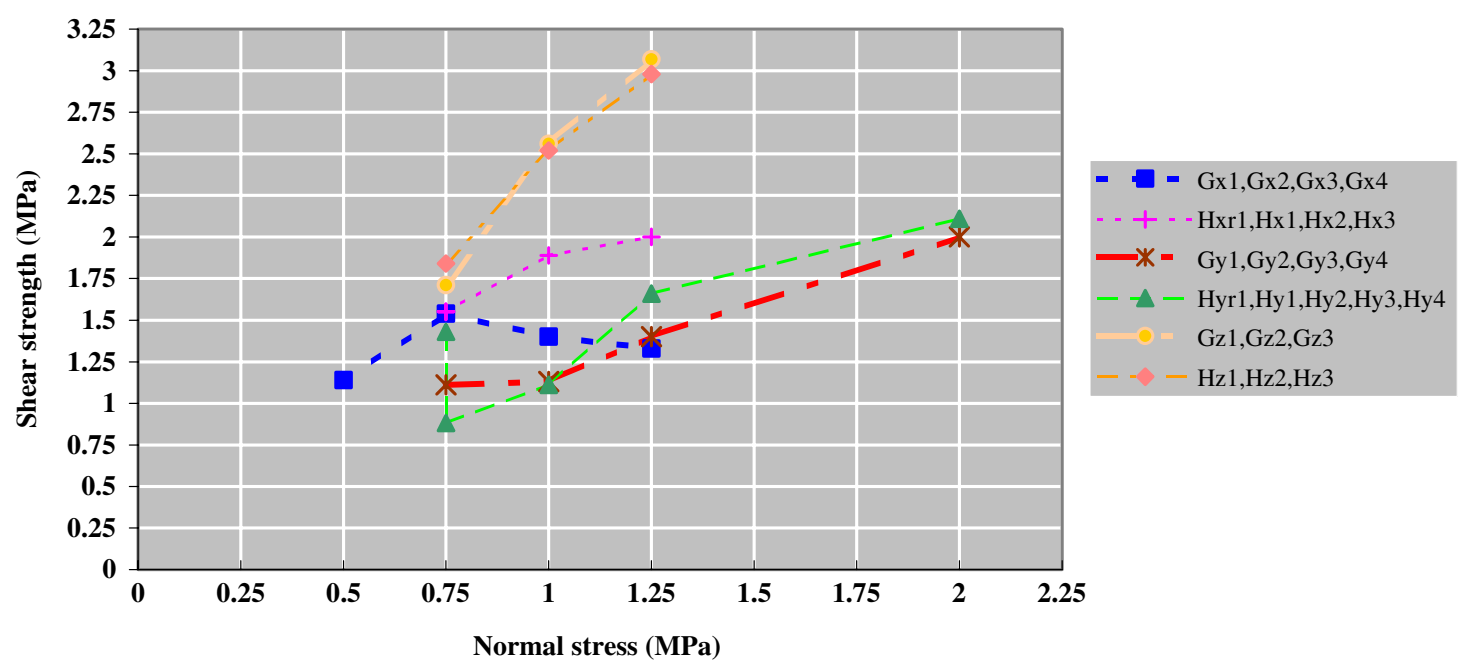

Fig. 8. Relation between the shear strength and the applied constant normal load in the shear tests (Gx, Hx, Gy, Hy, Gz, Hz series).

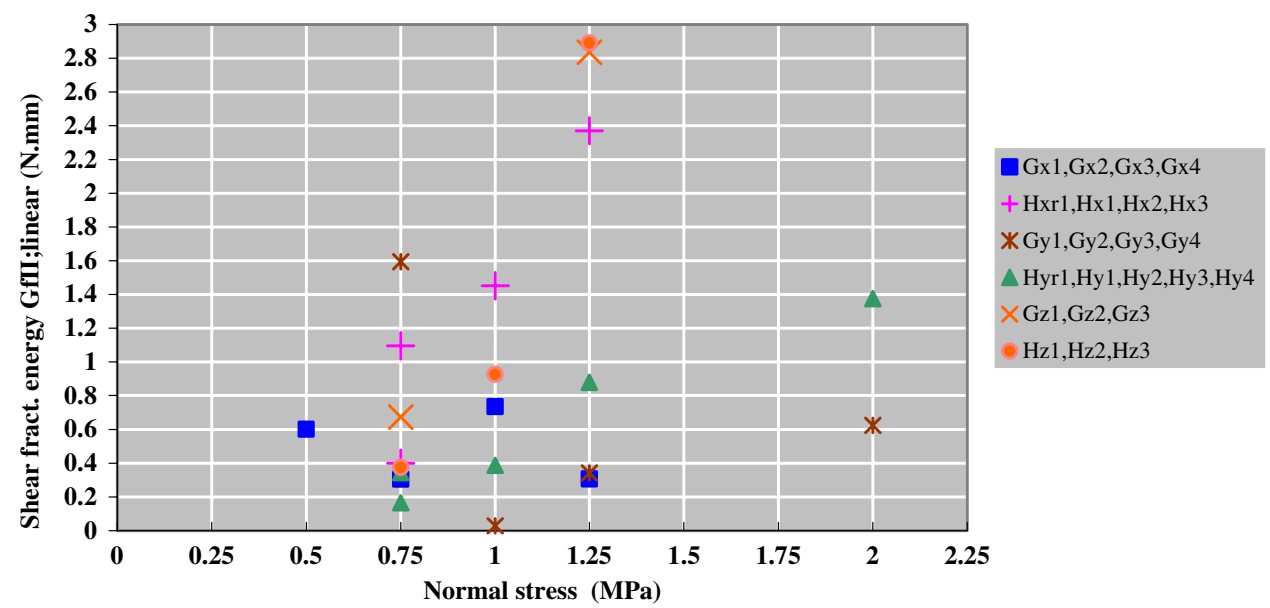

Fig. 9. Shear fracture energy versus normal applied stress for series Gx, Hx, Gy, Hy, Gz, Hz and additional specimens Hxr1 and Hyr1 (note that, for the same normal stress, GfII (Hxr1) < GfII (Hx1): and GfII (Hyrl) > GfII (Hyl)). 


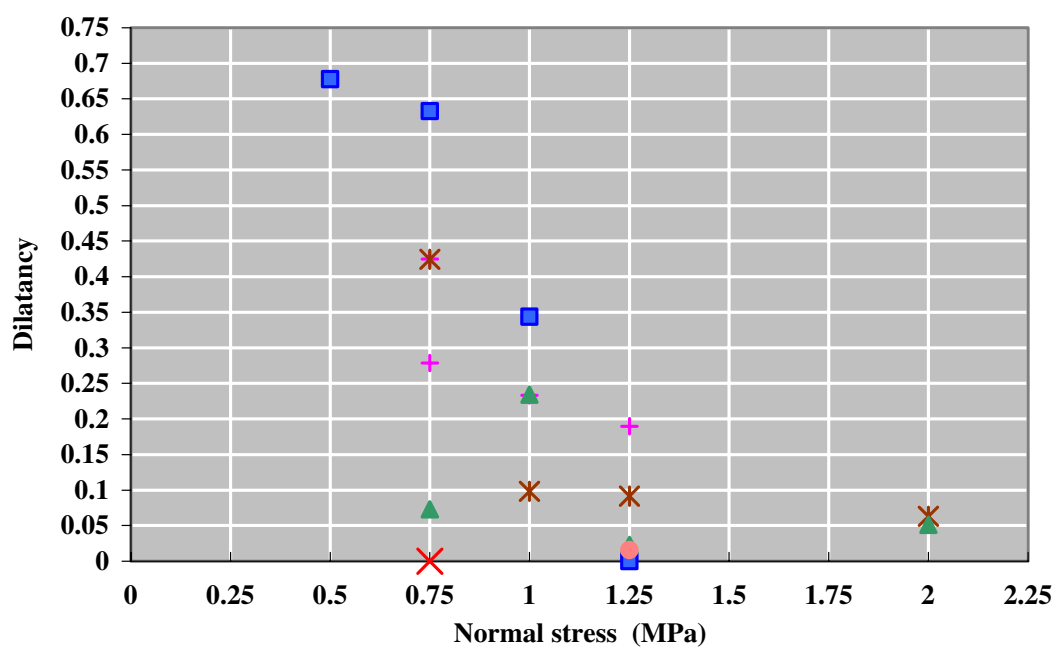

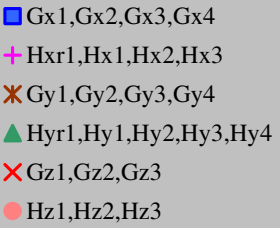

Normal stress (MPa)

Fig. 10. Tangent of dilatancy angle $\psi$ (dilatancy) versus normal applied stress for series Gx, Hx, Gy, Hy, Gz, Hz and additional specimens Hxr1 and Hyr1.

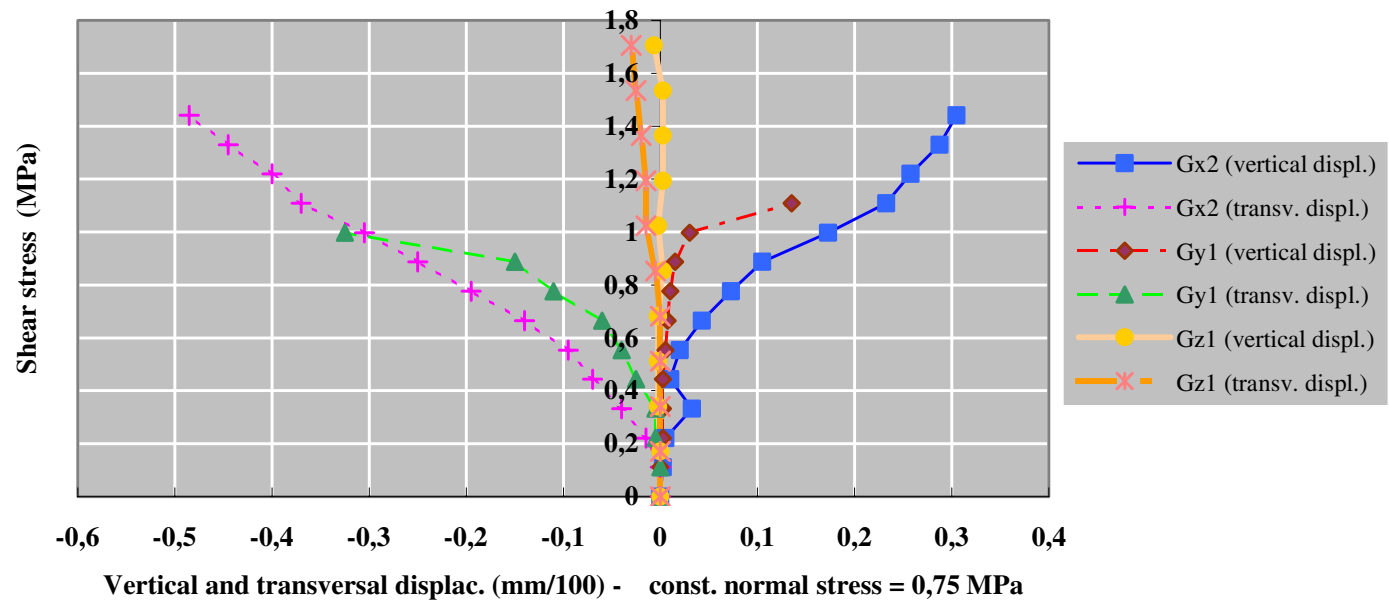

Fig. 11. Peak shear strength versus average shear displacements of Gx2, Gy1 and Gz1 specimens, during the shear test (normal constant stress $\sigma_{\mathrm{n}}=0.75 \mathrm{MPa}-$ specimen with simple horizontal joint).

$\mathrm{Hz}$ series, generally, produced minor changes, in terms of joint shear resistance of the specimens, when compared with the correspondent values in Gx, Gy and Gz series. For the same type of specimen and level of normal applied load $(0.75 \mathrm{MPa})$, shear and normal displacements varied substantially during the shear test (with increasing shear applied load) according to the type of material (decreasing of deformations from Gx2 to Gy1 and Gy1 to Gz1 Fig. 11). As was expected, an increase of joint peak shear strength, for each of those series, was generally detected with the increase of normal applied stress. An approximate linear relationship (Coulomb law) could be established between normal applied stress and joint peak shear strength for low levels of normal applied force in the specimens, but the limited number specimens in each series does not consent representative and definite conclusions. The friction coefficient and the cohesion values of $\mathrm{Gx}$ and $\mathrm{Hx}$ series are not significantly different. The inclusion of a metal expanded meshwork in the joints apparently maintained roughly the peak and residual shear strength values of those joints $(\mathrm{Gx} 2$ versus $\operatorname{Hxr} 1)$, or even increased them slightly (Gyl versus Hyr1). But the improvement of the deformation behaviour was detected from the recorded peak and residuals shear deformations especially in Hyr1.

While the shear peak value of Bk series was, generally lower then those of Gx series (similar block material), the shear stiffness was higher then those of Gx series, particularly near failure. Those results suggest that for low values of shear load, the block/concrete joint specimens was less resistant than block/block joint specimens. The inclusion of reinforcement in the joint, in case the specimen Bkrl $\left(\sigma_{\mathrm{n}}=0.75 \mathrm{MPa}\right)$, improved significantly the shear peak value when compared with specimen Bk1.

Comparing shear stiffness of specimens Bk2, Bkr1, Hxr1, Gx2, for low values of shear load, the mixed specimens (Bk2, Bkr1) show higher values than those of Hxr1, $\mathrm{Gx} 2$, but near failure the difference between them were less important. The same pattern of behavior can be found comparing shear stiffness of specimens Bw1, Bwrl to the ones of specimens Hyr1 and Gyl. 
The inclusion of reinforcement in the joint, in case the specimen Bwr1 $\left(\sigma_{\mathrm{n}}=0.75 \mathrm{MPa}\right)$ has not changed significantly the shear peak value when compared with specimen Bw1.

Comparing the shear tests results for material X, Y and $\mathrm{Z}$, with the shear strength values for walls with completely mortar filled joints tabulated in EC6 (EC6, [1], Table 3.5) it is possible to find that the previous values are generally higher that those of EC6 (note that for EC6 the characteristic shear strength value $f_{\mathrm{vk}}=f_{\mathrm{vko}}+0.4 . \sigma_{\mathrm{d}}$; being $\tan (\varphi)=0.4$ - value much lower than those obtained for Gx and Gy series - Tables 3 and 4). For clay brick or lightweight blocks used in the present study (units corresponding in EC6 to the group 1) and mortar M1 to M2 (for these type of mortar, $\mathrm{EC} 6 \Rightarrow f_{\mathrm{vko}}=0.1$ and limiting $\left.f_{\mathrm{vk}}=1.2\right)$, the values $f_{\mathrm{vk}}$ for normal stress of 0.5 and $1.25 \mathrm{MPa}$ (range of values common in the present study) are, respectively, 0.3 and $0.6 \mathrm{MPa}$, which, compared with values of Fig. 3, suggests that EC6 could be on the safe side, in the assessment of the friction coefficient and the cohesion values and, subsequently, the characteristic shear strength value.

Finally, from the results of the experimental tests it can be concluded that the properties of the referred block/ block and block/concrete joints are significant for the wall overall behavior as well as for the interaction between wall and concrete elements along their the interface, and the inclusion of reinforcing elements in these joints may, in some cases, determine positive implications.

\section{Final considerations}

The results of shear tests suggest that the mortar joint shear strain, at shear-stress level, can then be estimated for the maximum load (through maximum shear displacement).

The results of this experimental study on materials $\mathrm{X}, \mathrm{Y}$ and $\mathrm{Z}$ suggests that if failure in the block/block and block/ concrete joints occurs under a combination of shear and low compressive stress, a shear bond failure can be considered and some residual capacity will remain in the joint. The results allow to suppose that the shear resistance can be assumed to reduce approximately to zero between the situation of high compressive stress and tensile stress. So, these results indicate that the joint elements have appreciable compression capacity (see, in Tables 3-5, values of maximum normal displacements) and low tensile capacity (obtained from flexure tensile strength of couplets), and a shear capacity, which is a function of the imposed compression and the bond strength. The nonlinear characteristics of this type of masonry made of materials $\mathrm{X}, \mathrm{Y}$ and $\mathrm{Z}$ result most probably from the local failure and slip that occur in the joints and the nonlinear deformation typical of the joints under shear and compression. So, these characteristics must be taken in account when estimating the mechanical parameters for numerical simulations of masonry wall behavior.

From the results of the experimental tests, it can be concluded that the properties of the referred joints are significant for the wall overall behavior as well as for the interaction between masonry and concrete elements (beams and lintels), and the inclusion of reinforcing elements in these joints may, possibly, in some cases, determine positive implications, particularly, on resistance and deformation behavior of the corresponded masonry walls.

\section{Acknowledgement}

LNEC Programmed Research has funded the present study. The assistance and help in the experimental tests of Mr. Adolfo Silva is gratefully acknowledged.

\section{References}

[1] European Committee for Standardization, CEN. Eurocode No. 6, Design of masonry structures, Part. 1-1. General rules for buildings, rules for reinforced and unreinforced masonry, CEN, prENV 1996-1$1 ; 1995$.

[2] CEN. Methods of tests for masonry, Part. 3. Determination of initial shear strength, CEN, prEN 1052-3; 1999.

[3] Atkinson RH, Amadei BP, Saeb S. Response of masonry bed joints in shear. J Struct Eng Proc ASCE 1989;115(9):2276-96.

[4] Miranda Dias JL. Composite action between lightweight concrete masonry blocks and their supporting beams. Ph.D. thesis, IST, LNEC, Portugal; 1997.

[5] Miranda Dias JL. Control of thermal and moisture deformations in building masonry walls and confining reinforced concrete elements. In: Proceedings of XXX IAHS, world congress on housing, "an interdisciplinary task", September 2002, vol. 3, Coimbra, Portugal; 2002a. p. 1481-6.

[6] Miranda Dias JL. Movements in masonry walls caused by temperature and moisture changes. In: Proceedings of 6th international masonry conference, London, United Kingdom; 2002a. p. 86-94.

[7] Miranda Dias JL. Cracking around the interface joint between masonry panels and their supporting reinforced concrete beams in buildings. In: Proceedings of 2 nd international structural engineering and construction conference, vol. I, University of Rome, Italy; 2003. p. $745-52$.

[8] Miranda Dias JL. Susceptibility for cracking of masonry mortar joints when subjected to compression. In: Proceedings of 10th Canadian Masonry symposium, University of Calgary, Alberta, Canada, S. 3b; 2005.

[9] Pluijm R. A shear behaviour of bed joints. Proceedings of 6th North American masonry conference, vol. 1. PA, USA: Drexel University; 1993. p. 125-36.

[10] Ghazalli MZ, Riddington RJ. Simple test method for masonry shear strength. Proc Institut Civil Eng Part 2 1988;85:567-74.

[11] Stockl S, Hofmann P, Mainz J. Comparative finite element evaluation of mortar joint shear tests. Masonry Int $\mathbf{J}$ 1990;3(3):101-4. 